\title{
Editorial: Recent Developments in Pipeline Geotechnics
}

Pipelines transport a variety of gasses and fluids across continents and oceans and traverse extremes of terrain and environments. This special issue presents invited papers addressing a variety of pipeline geotechnics challenges both onshore and offshore, in a range of geotechnical conditions including arctic environments. The authors of the selected papers, all recognised experts in the field of pipeline geotechnics, are academicians and practitioners from Canada, USA, Europe, India, China and Australia.

Canadian Geotechnical Journal has traditionally served as a forum for presenting developments in geotechnical aspects of pipeline engineering. Over 100 articles on soil resistance developing during relative soil-pipeline movements, pipelines in permafrost, interaction of partially-embedded offshore pipelines with the seabed during heating-cooling episodes amongst other subjects have been published in Canadian Geotechnical Journal since 1972, with almost half of these published during the last 10 years - signifying an increasing interest in the subject. With Canadian Geotechnical Journal being a key journal for the publication of research findings and practical applications of pipeline geotechnics, it seemed natural to invite some of the leading groups from Canada and internationally to showcase their latest work in a special issue. The collection of articles covers active research areas and state-of-the-art practices in the design and analysis of new pipelines, and the evaluation of the performance of existing pipelines.

In the first part of the issue research groups and practitioners discuss noteworthy advances in soilburied pipeline and soil-culvert interaction problems. Using finite element analyses benchmarked against large-scale tests, Jai Jung from Youngstown State University together with Thomas O'Rourke and Christina Argyrou from Cornell University generalise the problem of estimating soil reaction to fully buried pipeline movements in dry or partially saturated sand, by considering arbitrary relative movement directions. Accurate quantification of soil-pipeline interaction is of prominent importance for the stress analysis of continuous and jointed pipelines against permanent ground deformations, with numerical or simplified methodologies such as that proposed for continuous buried pipelines with bends by Dimitrios Karamitros from the University of Bristol in collaboration with Christos Zoupantis and George Bouckovalas from the National Technical University of Athens, Greece. The assessment of the capacity of jointed pipelines such as cast iron pipes connected with lead-caulked bell and spigot joints used in pre-1960 networks, and modern ductile iron pipes with push-on joints, is of prominent importance for estimating the resilience of existing and new networks; this is examined by Brad Wham, Christina Argyrou and Thomas O'Rourke from the Cornell research group for urban networks overlying tunnelling works. Moreover, field inspection data and earth pressure measurements presented by Benjamin McGuigan from GEMTEC Limited in collaboration with industry colleagues Olajide Oshati from AECOM and Bethanie Parker from HATCH as well as Arun Valsangkar from the University of New Brunswick provide valuable information on the long-term performance of circular and box culverts constructed with the induced trench method.

The latter part of the issue addresses challenges in offshore pipeline geotechnics, from those relevant to shallow water sediments to deepwater frontiers. A selection of practitioners and academicians present their latest experiences and research. Fuping Gao, Ning Wang and Xi-Ting Han from the Chinese Academy of Sciences, in conjunction with colleague Jinhui Li from Harbin University, China present solutions for current induced lateral instability of submarine pipelines. Bruno Stuyts and David Cathie of Cathie Associates along with industry colleague Toby Powell of Subsea7 present a study of model uncertainty in uplift resistance calculations for sand backfills. Shubhrajit Maitra, Santiram Chatterjee and Deepankar Choudhury from the Indian Institute of Technology Bombay present a generalised framework to predict undrained uplift capacity of buried offshore pipelines; and a collaboration of industry and academic authors, Zach Westgate and William Haneberg of Fugro and David White of the Centre for Offshore Foundation Systems at the 
University of Western Australia present a study modelling the spatial variability of as-laid embedment for HPHT pipeline design.

Finally, Kenton Pike and Shawn Kenny from the Memorial University of Newfoundland and Carleton University, present engineering tools for the design of onshore and offshore pipelines in arctic environments.

Of course pipeline geotechnics is an extremely broad topic, and it is impossible include within the limited scope of a single journal issue, contributions representing all geotechnical aspects of pipeline geotechnics, or adequately represent the wealth of research and state-of-the art practice being carried out and applied by the many research groups and practitioners worldwide.

Canadian Geotechnical Journal continues to welcome contributions on all aspects of pipeline geotechnics, with the aim to remain at the forefront of publishing pipeline geotechnics research and its applications in pipeline engineering practice.

George Kouretzis and Susan Gourvenec,

Associate Editors of the Canadian Geotechnical Journal and invited Editors of this special issue. 